\title{
Dual antiplatelet therapy does not scare away the erector spinae plane block
}

\author{
Christopher A. Smith and Kelly M. Martin \\ Department of Anesthesia, Anesthesia Associates of York, WellSpan York Hospital, York, PA, USA
}

It is not unusual for readers of an article to have differing opinions. After reading the case report entitled "Erector spinae plane block combined with a novel technique for selective brachial plexus block in breast cancer surgery" by De Cassai et al. [1] and the letter to the editor entitled "The risks associated with erector spinae plane block in patients with abnormalities of coagulation" by Nair and Seelam [2] we feel compelled to respond.

Although we find value in Nair and Seelam's comments, their assertions that the medial and lateral pectoral, long thoracic and thoracodorsal nerves need not be blocked for a mastectomy should have been made with caveats [2]. First, the authors refer to these nerves as being "essentially motor nerves." To our knowledge, their role in nociception has not been well established; however they carry nociceptive fibers and are thought to contribute to pain related to the disruption, stretching or spasming of muscles or associated fascia [3]. This is an important point when chastising De Cassai et al. for their clinical decision to use a combined regional anesthesia technique to ensure the patient's comfort and a still operative field for the surgeon. Additionally, the authors group the intercostal nerves and the aforementioned nerves collectively as "anterior chest wall" innervation. It is important to remember that there are essentially two areas of innervation that require consideration in the planning of performing regional anesthesia for breast surgery: (1) the

Corresponding author: Christopher A. Smith, DNP, CRNA

Department of Anesthesia, Anesthesia Associates of York, WellSpan York Hospital, 1001 South George Street, 110 Pine Grove Commons, York, PA 17403, USA

Tel: 1-717-851-2462, Fax: 1-717-851-5250

Email: csmith21@wellspan.org

ORCID: https://orcid.org/0000-0002-7816-3016

Received: February 2, 2019.

Revised: February 17, 2019.

Accepted: February 18, 2019.

Korean J Anesthesiol 2019 June 72(3): 277-278

https://doi.org/10.4097/kja.19044 cutaneous distribution which is innervated by intercostal nerves II-VI as well as the supraclavicular nerve branches from the cervical plexus; and (2) the anterior chest wall which is innervated by the aforementioned branches of the brachial plexus as well as the intercostal nerves. Therefore, the regional anesthesia technique (s) should match that of the surgical procedure and cover the area (s) being violated or manipulated (i.e., simple mastectomy versus reconstructive breast surgery).

Our next point of contention arises from Nair and Seelam's assertion that the erector spinae plane (ESP) block is a deep, perineuraxial block [2]. This claim was made without reference or corroboration searching within (https://www.nysora.com); (https://www.asra.com); (https://esraeurope.org). In regard to anticoagulation, American Society of Regional Anesthesia and Pain Medicine guidelines "suggest management based on site compressibility, vascularity, and consequences of bleeding should it occur" [4]. To that point, Chin et al. [5] describe the ESP as being remote to major nervous and vascular structures thereby minimizing risks of clinically significant hematomas in anticoagulated patients. The erector spinae muscles can be compressed in the unlikely event of hematoma formation; however, to date, there are no reported cases in the literature [2].

Anesthetic choices should be made after weighing the risks and benefits for the patient based upon pathophysiology, mutual outcome goals (surgeon, patient and anesthesia provider) and patient preferences. We believe that De Cassai et al. [1] presented an interesting and effective approach for avoiding general anesthesia in a patient with significant comorbidities.

\section{Acknowledgments}

WellSpan Library services staff for their continued support.

\section{Conflicts of Interest}

No potential conflict of interest relevant to this article was

(c) This is an open-access article distributed under the terms of the Creative Commons Attribution Non-Commercial License (http://creativecommons.org/ licenses/by-nc/4.0/), which permits unrestricted non-commercial use, distribution, and reproduction in any medium, provided the original work is properly cited. 
reported.

\section{Author Contributions}

Christopher A. Smith (Writing - original draft)

Kelly M. Martin (Writing - original draft)

\section{ORCID}

Christopher A. Smith, https://orcid.org/0000-0002-7816-3016

Kelly M. Martin, https://orcid.org/0000-0003-3511-4764

\section{References}

1. De Cassai A, Bonvicini D, Ruol M, Correale C, Furnari M. Erector spinae plane block combined with a novel technique for selective brachial plexus block in breast cancer surgery. Korean J Anesthesiol 2019; 72: 270-4.

2. Nair AS, Seelam S. The risks associated with erector spinae plane block in patients with abnormalities of coagulation. Korean J Anesthesiol 2019; 72: 275-6.

3. Woodworth GE, Ivie RM, Nelson SM, Walker CM, Maniker RB. Perioperative breast analgesia: a qualitative review of anatomy and regional techniques. Reg Anesth Pain Med 2017; 42: 609-31.

4. Horlocker TT, Vandermeuelen E, Kopp SL, Gogarten W, Leffert LR, Benzon HT. Regional Anesthesia in the Patient Receiving Antithrombotic or Thrombolytic Therapy: American Society of Regional Anesthesia and Pain Medicine Evidence-Based Guidelines (Fourth Edition). Reg Anesth Pain Med 2018; 43: 263-309.

5. Chin KJ, Malhas L, Perlas A. The erector spinae plane block provides visceral abdominal analgesia in bariatric surgery: a report of 3 cases. Reg Anesth Pain Med 2017; 42: 372-6. 